\title{
Incidental Finding
}

National Cancer Institute

\section{Source}

National Cancer Institute. Incidental Finding. NCI Thesaurus. Code C48619.

An asymptomatic benign or malignant lesion discovered during an endoscopic, surgical,

or radiologic procedure performed for unrelated reasons. 